\title{
Valoración de la relación entre la restricción rotacional de columna cervical alta y columna torácica alta en alumnas con y sin dolor cervical de la Facultad de Ciencias de la Rehabilitación de la Universidad Nacional Andrés Bello-Campus Casona
} Valuation of the relationship between the rotational restriction of high cervical column and high thoracic column in students with and without cervical pain of the Faculty of Sciences of the Rehabilitation of the National University Andrés Bello-Campus Casona

R. Guamaní Vásquez y D. Gatica

Pontificia Universidad Católica del Ecuador. Quito, Ecuador

\section{ABSTRACT}

Introduction: Cervical pain is one of the disorders that most affects and worries people. One of its possible causes are joint dysfunctions that can develop in the upper cervical, lower cervical and upper thoracic spines. However, studies that have focused on the dysfunctional relationships of both columns in a transverse plane are still very scarce.

Objective: To contribute to the knowledge about cervical pain and clinical analysis, the objective of this study was to evaluate if there is a relationship between the rotational mobility restriction of the high cervical spine (C1-C2) and the high thoracic spine (T1-T2-T3) in female students with and without cervical pain.

Material and methodology: 37 female students with acute neck pain and 37 asymptomatic female students belonging to the Faculty of Rehabilitation Sciences of the Andrés Bello University were recruited. The rotational mobility of the upper cervical spine was evaluated by means of the Dvorák Flexion-Rotation Test and the rotational mobility of the upper thoracic spine was evaluated with the Mitchell Test.

\section{RESUMEN}

Introducción: El dolor cervical es uno de los trastornos que más afecta y preocupa a las personas. Una de sus posibles causas son las disfunciones articulares que pueden desarrollarse en la columna cervical alta, cervical baja y torácica alta. Sin embargo, los estudios que se han enfocado en las relaciones disfuncionales de ambas columnas en un plano transverso aún son muy escasos.

Objetivo: Para contribuir al conocimiento sobre dolor cervical y al análisis clínico, se planteó como objetivo de este estudio evaluar si existe relación entre la restricción de movilidad rotacional de columna cervical alta (C1-C2) y columna torácica alta (T1-T2-T3) en alumnas con y sin dolor cervical.

Material y metodología: Se reclutaron 37 alumnas con dolor cervical agudo y 37 alumnas asintomáticas pertenecientes la Facultad de Ciencias de la Rehabilitación de la Universidad Andrés Bello. La movilidad rotacional de columna cervical alta se evaluó mediante el Test de Flexión-Rotación de Dvorák y la movilidad rotacional de columna torácica alta fue evaluada con el Test de Mitchell.

Recibido: 10-07-2018

Aceptado: 31-10-2019

Correspondencia: Rodrigo Guamaní Vásquez romaster1985@gmail.com
R. Guamaní Vásquez y D. Gatica. Valoración de la relación entre la restricción rotacional de columna cervical alta y columna torácica alta en alumnas con y sin dolor cervical de la facultad de ciencias de la rehabilitación de la Universidad Nacional Andrés Bello-Campus Casona Rev Soc Esp Dolor 2019;26(6):337-341 
Results and conclusions: Based on the results obtained, it was concluded that there was no relationship between cervical pain, rotational restriction of the upper and upper thoracic spine. In addition, subsequent studies are necessary to have a better understanding of the functional relationship of these segments.

Key words: Column, pain, neck, mobility restriction, women.
Resultados y conclusiones: En base a los resultados obtenidos se pudo concluir que no hubo relación entre el dolor cervical, restricción rotacional de columna cervical alta y torácica alta. Además, estudios posteriores son necesarios para tener un mejor entendimiento de la relación funcional de dichos segmentos.

Palabras clave: Columna, dolor, cuello, restricción de movilidad, mujeres.

\section{INTRODUCCIÓN}

El dolor cervical es una de las afecciones musculo-esqueléticas con mayor prevalencia, que tiene como resultado limitaciones físicas y funcionales (1). Como resultado, numerosos estudios se han enfocado solo en el análisis de las disfunciones de la columna cervical, sin considerar que las disfunciones de la columna torácica pueden influir también en la aparición del dolor cervical. En consecuencia, actualmente existe escasa información sobre cómo la columna cervical puede estar afectada por la falta o exceso de movilidad de la columna torácica alta. No obstante, se ha podido comprobar que la lordosis cervical se ve influenciada por la angulación sagital del tórax y del complejo articular cérvico torácico [2]. Por otro lado, la restricción de movilidad de la columna cervical se ha asociado con la hipomovilidad en escoliosis torácica (3), lo cual sugiere una vinculación entre las vértebras cervicales y torácicas en un plano sagital como frontal (1). Sin embargo, la relación de movilidad existente en un plano transversal aún no ha sido del todo esclarecida. Para tener un mejor entendimiento de esta relación, el objetivo del presente estudio fue valorar la relación entre la columna cervical alta y torácica alta en uno de los problemas más comunes de la práctica kinésica, como lo es el dolor cervical. Debido a que se ha evidenciado que las mujeres tienden a desarrollar dolor de cervical con mayor frecuencia en comparación con hombres $(4,5)$, este estudio solo se centró en mujeres.

\section{MATERIALES Y METODOLOGÍA}

De las alumnas pertenecientes a la Facultad de Ciencias de la Rehabilitación de la Universidad Nacional Andrés Bello-Campus Casona, se reclutaron 74 alumnas para ser evaluadas en este estudio.

Los datos obtenidos tras las evaluaciones de las alumnas fueron registrados en fichas elaboradas por los autores, las cuales, en una primera instancia, fueron confeccionadas mediante el programa Microsoft Excel 2013 para el análisis de las variables. Posteriormente se traspasaron los datos al programa G-Stat, en el cual, mediante la prueba de Chi cuadrado, se evaluó la relación existente entre las variables independientes, cuantitativas y paramétricas.
De acuerdo con los resultados obtenidos tras la aplicación de la prueba Chi cuadrado, si el valor de $p$ encontrado era mayor a 0,05, el resultado se interpretaba como no significativo y se concluía que ambas variables estudiadas eran independientes la una de la otra. Por el contrario, si el valor de p encontrado era menor a 0,05, el resultado era interpretado como significativo y se concluía que ambas variables estudiadas eran dependientes entre ellas.

Al total de 74 alumnas, divididas en dos subgrupos de 37 con dolor cervical y 37 sin dolor cervical, se les realizó el Test de Flexión-Rotación de Dvorák con el fin de evaluar la restricción de movilidad de columna cervical alta, y el Test de Mitchell para evaluar la restricción de movilidad de columna torácica alta.

La recolección de datos se llevó a cabo en el mes de mayo del año 2017.

Todas las alumnas fueron enroladas en el estudio de forma voluntaria y firmaron un consentimiento informado diseñado por los evaluadores.

Para llevar a cabo este estudio se utilizaron las instalaciones de la Facultad de Ciencias de la Rehabilitación de la Universidad Andrés Bello-Campus Casona y una camilla regulable en altura, perteneciente a la misma institución.

\section{Escala de Evaluación Visual Análoga del Dolor [EVA]}

Ventajas de esta escala son su simplicidad, su uniformidad y su confiabilidad (6).

Para medir la intensidad del dolor se requirió que la alumna marcara un punto en la línea que refleja la cantidad de dolor que presentó en ese momento entre "no dolor" y "peor dolor que haya sentido".

\section{Test de Mitchell}

El Test de Mitchell posee un $82 \%$ de sensibilidad y un $79 \%$ de especificidad (7).

\section{Test de Flexión-Rotación de Dvorák}

El Test de Flexión-Rotación de Dvorák posee un 91 \% de sensibilidad y un $90 \%$ de especificidad (8). 


\section{RESULTADOS DE LA INVESTIGACIÓN}

En la evaluación realizada a 74 alumnas divididas en dos subgrupos de 37 alumnas, conformados por aquellas con dolor cervical agudo y por aquellas sin dolor cervical, se revelaron los siguientes resultados:

- En el subgrupo que no presentó dolor cervical y que se evaluó la restricción de movilidad rotacional de C1-C2, se detectó que 19 alumnas presentaron restricción rotacional a la derecha, 8 presentaron restricción rotacional a la izquierda mientras que 10 no presentaron restricción rotacional.

- En el subgrupo con dolor cervical se detectó que 23 alumnas presentaron restricción rotacional derecha, 8 presentaron restricción rotacional a la izquierda mientras que 6 no presentaron restricción rotacional.

Tras aplicar la prueba de Chi cuadrado para analizar la relación existente entre las variables restricción de movilidad rotacional de C1-C2, dolor y no dolor cervical, se obtuvo un valor de $p$ mayor a 0,05 , por lo tanto se interpretó como ausencia de relación entre las variables categorizadas.

Con respecto a los resultados de la evaluación de la restricción de movilidad rotacional de los segmentos T1, T2 y T3 en alumnas con y sin dolor cervical agudo, se pudo observar lo siguiente: en el subgrupo en donde se evaluó la presencia de restricción de movilidad rotacional de T1 en alumnas sin dolor cervical se detectó que una alumna presentó restricción rotacional a la derecha, 14 presentaron restricción rotacional a la izquierda, mientras que 22 no presentaron restricción rotacional. En el subgrupo en donde se evaluó la presencia de restricción de movilidad rotacional de T1 en alumnas con dolor cervical agudo se detectó que 9 alumnas presentaron restricción rotacional de la derecha, 23 presentaron restricción rotacional a la izquierda, mientras que 5 no presentaron restricción rotacional. Tras aplicar la prueba de Chi cuadrado para analizar la relación existente entre las variables restricción de movilidad rotacional de T1, dolor y no dolor cervical, se obtuvo un valor de $p$ menor a 0,05, lo cual se interpretó como existencia de relación entre las variables categorizadas.

En relación con la restricción de movilidad rotacional de T2 en el subgrupo de alumnas sin dolor cervical, se observó que 2 alumnas presentaron restricción rotacional a la derecha, 16 presentaron restricción rotacional a la izquierda mientras que 19 no presentaron restricción rotacional.

En relación con la restricción de movilidad rotacional de T2 en el subgrupo de alumnas con dolor cervical agudo, se observó que 10 alumnas presentaron restricción rotacional a la derecha, 23 presentaron restricción rotacional a la izquierda, mientras que 4 no presentaron restricción rotacional. Tras aplicar la prueba de Chi cuadrado para analizar la relación existente entre las variables restricción de movilidad rotacional de T2, dolor y no dolor cervical, se obtuvo un valor de $p$ menor a 0,05 , lo cual se interpretó como existencia de relación entre las variables categorizadas.

En relación con la restricción de movilidad rotacional de T3 en alumnas sin dolor cervical, se observó que 2 alumnas presentaron restricción rotacional a la dere- cha, 14 presentaron restricción rotacional la izquierda, mientras que 21 no presentaron restricción rotacional.

En relación con la restricción de movilidad rotacional de T3 en alumnas con dolor cervical agudo, se observó que 6 alumnas presentaron restricción rotacional a la derecha, 22 presentaron restricción rotacional a la izquierda, mientras que 9 no presentaron restricción rotacional. Tras aplicar la prueba de Chi cuadrado para analizar la relación existente entre las variables restricción de movilidad rotacional de T3, dolor y no dolor cervical, se obtuvo un valor de $p$ menor a 0,05, lo cual se interpretó como existencia de relación entre las variables categorizadas.

Con respecto a la relación que hay entre la restricción de movilidad rotacional de C1-C2 y restricción de movilidad rotacional de T1, se observó lo siguiente: de un total de 58 alumnas que presentaron restricción rotacional de C1-C2, 5 alumnas presentaron restricción rotacional de C1-C2 a la derecha con restricción rotación de T1 a la derecha, 20 presentaron restricción rotacional de C1-C2 a la derecha con restricción rotacional de T1 a la izquierda, 3 presentaron restricción rotacional de C1-C2 a la izquierda con restricción rotacional de T1 a la derecha, 7 presentaron restricción rotacional de C1-C2 a la izquierda con restricción rotacional de T1 a la izquierda mientras que 17 alumnas que presentaron restricción rotacional de C1-C2 a la derecha y 6 que presentaron restricción rotacional de C1-C2 a la izquierda, no presentaron restricción rotacional de T1.

Tras aplicar la prueba de Chi cuadrado para analizar la relación existente entre las variables de restricción de movilidad rotacional de C1-C2 con restricción de movilidad rotacional de T1, se obtuvo un valor de $p$ mayor a 0,05, lo cual se interpretó como ausencia de relación entre las variables categorizadas.

Con respecto a la relación que hay entre la restricción de movilidad rotacional de C1-C2 y restricción de movilidad rotacional de T2, se observó lo siguiente: de un total de 58 alumnas que presentaron restricción rotacional de C1-C2, 5 alumnas presentaron restricción rotacional de C1-C2 a la derecha con restricción rotación de T2 a la derecha, 21 presentaron restricción rotacional de C1-C2 a la derecha con restricción rotacional de T2 a la izquierda, 3 presentaron restricción rotacional de C1-C2 a la izquierda con restricción rotacional de T2 a la derecha, 8 presentaron restricción rotacional de C1-C2 a la izquierda con restricción rotacional de T2 a la izquierda mientras que 16 alumnas que presentaron restricción rotacional de C1-C2 y 5 que presentaron restricción rotacional a la izquierda de C1-C2, no presentaron restricción rotacional de T2. Tras aplicar la prueba de Chi cuadrado para analizar la posible relación existente entre las variables de restricción de movilidad rotacional de C1-C2 con restricción de movilidad rotacional de T2, se obtuvo un valor de $p$ mayor a 0,05, por lo tanto se interpretó como ausencia de relación entre las variables categorizadas.

Con respecto a la relación que hay entre la restricción de movilidad rotacional de columna cervical alta y restricción de movilidad rotacional de T3, se observó lo siguiente: de un total de 58 alumnas que presentaron restricción rotacional de C1-C2, 2 alumnas presentaron restricción rotacional de C1-C2 a la derecha con 
restricción rotación de T2 a la derecha, 23 presentaron restricción rotacional de C1-C2 a la derecha con restricción rotacional de T2 a la izquierda, 4 presentaron restricción rotacional de C1-C2 a la izquierda con restricción rotacional de T2 a la derecha, 9 presentaron restricción rotacional de C1-C2 a la izquierda con restricción rotacional de T2 a la izquierda mientras que 17 alumnas que presentaron restricción rotacional de C1-C2 y 3 que presentaron restricción rotacional la izquierda de C1-C2, no presentaron restricción rotacional de T2. Tras aplicar la prueba de Chi cuadrado para analizar la relación existente entre las variables de restricción de movilidad rotacional de C1-C2 con restricción de movilidad rotacional de T3, se obtuvo un valor de $p$ mayor a 0,05 , lo cual se interpretó como ausencia de relación entre las variables categorizadas.

\section{DISCUSIÓN}

En este estudio se investigó la relación que hay entre el dolor cervical, la restricción de movilidad rotacional de columna cervical alta y la restricción de movilidad rotacional de columna torácica alta. Según nuestros resultados que relacionaron la presencia o ausencia de dolor cervical con la presencia o ausencia de restricción de movilidad rotacional de columna cervical alta, se pudo apreciar que hubo ausencia de relación; esto quiere decir que, ante la presencia de dolor cervical, no necesariamente nos encontraremos con restricción de movilidad rotacional de columna cervical alta. Por otro lado, se ha descrito que la disminución de la movilidad cervical ante la presencia de dolor cervical puede deberse a mecanismos neurofisiológicos de origen central desarrollados por la persona afectada que, por una parte, provocarían miedo y rechazo al movimiento cervical y, por otra, cambios estructurales y funcionales en la musculatura perteneciente a la columna cervical que limitarían el movimiento [9].

La restricción de movilidad rotacional de C1-C2 no necesariamente causó dolor cervical y pérdida de la funcionalidad cervical, por lo tanto, en relación con nuestros resultados, si evaluamos a una persona que presente restricción de movilidad en un segmento en particular, no necesariamente tendrá dolor y disfunciones musculares en la zona correspondiente a ese segmento.

La existencia de dolor sí tuvo relación con la presencia de restricción de movilidad rotacional en los segmentos T1, T2 y T3; este es un punto muy importante a tener en cuenta, ya que se ha postulado que los pacientes que han recibido manipulación de columna torácica, experimentaron una mayor disminución en el dolor cervical en relación a quienes no recibieron manipulación de la misma (10). Esto podría ser un indicativo de que la restricción de movilidad de columna torácica alta sea una compensación para evitar el aumento de dolor cervical, pero que en el corto o largo plazo podría aumentarlo, o bien la restricción de movilidad rotacional de columna torácica alta podría causar disfunciones articulares cervicales y que estas últimas causen el dolor cervical.

Respecto a la relación entre restricción de movilidad rotacional de columna cervical alta y torácica alta se pudo apreciar que no se encontró relación alguna, independientemente de si la persona tuvo o no dolor cervical, por lo tanto nuestra hipótesis planteada no se pudo cumplir. Esto nos podría dar un indicio de que la falta de movilidad rotacional de columna cervical alta no necesariamente podría provocar disfunciones en la columna torácica alta. Es importante destacar que el segmento C1-C2, por sí solo, aporta el $50 \%$ de la movilidad rotacional de la columna cervical (11), y que la manipulación de este puede mejorar el rango de movimiento rotacional cervical, si es que se presenta una disminución de movimiento en el plano transverso. Por otro lado, la manipulación torácica puede disminuir el dolor cervical y mejorar el rango de movimiento cervical $(12,13)$. En este punto se podría pensar que ambos segmentos vertebrales son funcional y directamente dependientes los unos de los otros, pero en base a nuestros resultados no se podría demostrar esto.

Hay que tener en cuenta que músculos como el esplenio del cuello, elevador de la escápula, romboides, trapecio, multífidos, semiespinoso de la cabeza y el cuello, entre otros, son músculos tónicos que unen la columna torácica con la columna cervical, lo que conllevaría a que ante la presencia de disfunciones musculares y/o articulares a nivel de los segmentos torácicos, pueda afectar a los segmentos cervicales debido a que la musculatura antes nombrada aumenta su tono y disminuye su longitud al presentar alguna disfunción (14), lo que podría rigidizar las zonas afectadas y traspasar las disfunciones presentes de una columna a otra.

En conclusión, existen diversas estructuras que unen directamente la columna cervical con la torácica; aun así, pese a existir esta unión, no se puede asegurar que si la columna cervical presenta una disfunción articular, la columna torácica también la desarrolle.

\section{BIBLIOGRAFÍA}

1. Tsang S, Szeto G, Lee R. Normal kinematics of the neck: The interplay between the cervical and thoracic spines. Man Ther. 2013;18(5):431-7. DOI: 10.1016/j.math.2013.03.002.

2. Hardacker JW, Shuford RF, Capicotto PN, Pryor PW. Radiographic standing cervical segmental alignment in adult volunteers without neck symptoms. Spine (Phila Pa 1976). 1997;22(13):1472-80. DOI: 10.1097/00007632$199707010-00009$

3. Floman Y. Thoracic scoliosis and restricted neck motion: a new syndrome? A report of six cases. Eur Spine J. 1998;7(2):155-7. DOl: 10.1007/s005860050046.

4. Bravo Acosta T. Diagnóstico y rehabilitación en enfermedades ortopédicas. La Habana: Editorial Ciencias Médicas; 2006. p. 149.

5. Palanca I, Puig R, Somoza J, Bernal J, Paniagua J. Unidad de tratamiento de dolor: estándares y recomendaciones. Madrid: Ministerio de Sanidad, Política Social e Igualdad; 2011. p. 13-39.

6. Serrano-Atero M, Caballero J, Cañas A, García P, SerranoÁlvarez C, Prieto J. Valoración del Dolor. Rev Soc Esp Dolor. 2002;9(2):94-108.

7. Cook C, Hegedus E. Orthopedic Physical Examination Tests: An Evidence-Based Approach. 2nd Edition; 2012. p. 140.

8. Ogince M, Hall T, Robinson K, Blackmore A. The diagnostic validity of the cervical flexion-rotation test in $\mathrm{C} 1 / 2$-related 
cervicogenic headache. Man Ther. 2007;12(3):256-62. DOI: 10.1016/j.math.2006.06.016.

9. Bahat H, Weiss P, Sprecher E, Krasovsky A, Laufer Y. Do neck kinematics correlate with pain intensity, neck disability or with fear of motion? Man Ther. 2014;19(3):252-8. DOI: 10.1016/j.math.2013.10.006

10. Salom M, Ortega S, Cleland J, Palacios C, Truyols D, Fernández de las Peñas C. Immediate changes in neck pain intensity and widespread pressure pain sensitivity in patients with bilateral chronic mechanical neck pain: a randomized controlled trial of thoracic thrust manipulation vs non-thrust mobilization. Journal of manipulative and physiological therapeutics. J Manipulative Physiol Ther. 2014;37(5):312-9. DOl: 10.1016/j.jmpt.2014.03.003.
11. Neumann D. Kinesiology of the Musculoskeletal System. 2nd ed. Mosby; 2009. p. 384-8.

12. Cleland J, Childs J, McRae M, Palmer J, Stowell T. Immediate effects of thoracic manipulation in patients with neck pain: a randomised clinical trial. Man Ther. 2005;10(2):127-35. DOl: 10.1016/j.math.2004.08.005.

13. Cross K, Kuenze C, Grindstaff T, Hertel J. Thoracic Spine Thrust Manipulation Improves Pain, Range of Motion, and Self-Reported Function in Patients With Mechanical Neck Pain: A Systematic Review. J Orthop Sports Phys Ther. 2011;41(9):633-42. DOI: 10.2519/jospt.2011.3670.

14. Janda V. Muscles, central nervous motor regulation and back problems. In: Korr IM. (eds). The Neurobiologic Mechanisms in Manipulative Therapy. Boston, MA: Springer; 1978. p. 27-41. 